\title{
TWO SPECIAL APPLICATIONS OF RIESZ PRODUCTS
}

\author{
MATS ERIK ANDERSSON \\ Matematiska inst., Stockholms universitet, SE-106 91 Stockholm, Sweden \\ E-mail:matsa@matematik.su.se
}

\begin{abstract}
Riesz products have been in use for constructing measures for a long time. Sometimes only a subset of the thus produced measures satisfy the desired properties. Here two such cases will be examined. The first pertains to the uniform integrability of partial sums and consists of examining the known three instances of Riesz-like products generating such measures.

The second - and more substantial part - investigates to what extent the Fourier-Stieltjes transform of a Riesz product defines a Hankel-Littlewood multiplier on the space $L\left(\ell^{2}(\mathbb{N})\right)$ of operators.
\end{abstract}

Controlling partial sums for Riesz products. It has long been known that any formal Fourier series $\nu \sim \sum c_{k} e^{i k \theta}$ with the property that the partial sums $s_{N} \nu=$ $\sum_{-N}^{N} c_{k} e_{k}$ are uniformly bounded in $L^{1}$-norm, must in fact be the Fourier-Stieltjes series of a measure $\nu \in M_{0}(\mathbb{T})$. For convenience the characters $e_{k}$ are defined by $e_{k}(\theta)=e^{i k \theta}$. The algebra $M_{0}(\mathbb{T})$ consists of all measures in the measure algebra $M(\mathbb{T})$, whose Fourier coefficients vanish at infinity. Let henceforth $M_{b}$ denote the vector space of all measures $\nu$ with $\sup _{N}\left\|s_{N} \nu\right\|_{1}$ finite. Clearly every $\nu \in M_{b}$ is a continuous measure.

The above result was achieved by Helson $[\mathrm{H}]$ and settled a conjecture by Steinhaus. The first to construct a singular measure in $M_{b}$ was Weiss [W]. Next, Katznelson [K] developed a variant with the added touch that all partial sums be positive. Later on also Brown and Hewitt $[\mathrm{Br}-\mathrm{H}]$ have given a general construction, producing singular measures with positive partial sums and prescribed decay of Fourier coefficients. As known to the present author, no further publication addresses the construction of measures with $L^{1}$-bounded partial sums.

It is the intent of this paper to display the fact that the above three papers produce measures in the radical $\operatorname{Rad} L^{1}$. More precisely the result is as follows.

SynOPSIS. The singular measures known in the literature to belong to $M_{b}$, do all have the property $\nu * \nu \in L^{2}(\mathbb{T})$.

2000 Mathematics Subject Classification: Primary 42A55; Secondary 42A16, 47L25.

Key words and phrases: Riesz product, radical, Schur multiplier, Littlewood function.

The paper is in final form and no version of it will be published elsewhere. 
The three published constructions demand separate handling, so are confined to one section each.

Analysis of Weiss' construction. The result of Weiss' deals with classical Riesz products and lacunary index sets. Recall that the original Riesz product concerns expressions

$$
\prod_{k=1}^{\infty}\left(1+a_{k} \cos n_{k} \theta\right),
$$

converging weak-* in $M(\mathbb{T})$. Here $-1 \leqslant a_{k} \leqslant 1$ and the integers $0<n_{1}<n_{2}<\ldots$ are lacunary in the sense $n_{k+1} / n_{k} \geqslant 3$. This is the setting for Weiss' contribution. General facts of relevance to Riesz products are found in the monograph by Graham and McGehee [G-M]. Later on, a generalised Riesz product will be described.

THEOREM $[\mathrm{W}]$. Let the real parameters $\left\{a_{k}\right\}$ of a Riesz product satisfy the condition

$$
\left|a_{k}\right|\left(\left|a_{1}\right|+\ldots+\left|a_{k}\right|\right)=\mathcal{O}(1) .
$$

Then the resulting measure belongs to $M_{b}$.

This result was originally applied with $a_{k}=1 / \sqrt{k}$, which clearly gives $\sum_{1}^{k} a_{j} \sim 2 \sqrt{k}$ and $\sum_{1}^{k} a_{j}^{2} \sim \log k$. As is well known [G-M, Thm. 7.2.1], the divergence expressed by the second relation makes the Riesz product a singular measure and it belongs to $M_{b}$ by Weiss' theorem.

It should be observed that the stronger decay $\left|a_{k}\right|\left(\left|a_{1}\right|+\ldots+\left|a_{k}\right|\right)=o(1)$ does not improve the conclusion as far as producing an absolutely continuous measure. This can be seen when studying $b_{k}=1 / \sqrt{k \log k}$, upon which $\sum_{1}^{k} b_{j} \sim 2 \sqrt{k} / \sqrt{\log k}$ and $\sum_{1}^{k} b_{j}^{2} \sim \log \log k$. Hence the resulting measure is still singular.

Proposition 1. Let the measure $\nu$ be constructed according to the preceding theorem. Then $\nu * \nu \in L^{2}(\mathbb{T})$ and $s_{N}(\nu * \nu) \rightarrow \nu * \nu$ in $L^{2}$ as well as in $L^{1}$. In contrast, $s_{N} \nu$ does not converge in $M(\mathbb{T})$, should $\sum a_{k}^{2}=\infty$ take place.

The last statement is clear, since the $L^{1}$-functions $s_{N} \nu$ tend weak-* to the singular measure $\nu$, whence no convergence in norm is possible. It thus suffices to demonstrate $\nu * \nu \in L^{2}$, from which the remaining claim follows. A simple case of real analysis prepares for this.

Lemma 2. Consider sequences $\left\{x_{k}\right\}_{1}^{\infty}$ of positive numbers, such that for all indices $k \geqslant 1$, the inequality $x_{k}\left(x_{1}+\ldots+x_{k}\right) \leqslant 1$ holds. For any such sequence and $p>2$, the series $\sum x_{k}^{p}$ converges.

Observe first that the already mentioned example $x_{k}=1 / \sqrt{2 k}$ shows the condition $p>2$ to be best possible.

Put now $X(k)=\left[x_{1}+\ldots+x_{k}\right]^{2}$. Clearly the two identities

$$
\begin{gathered}
X(k)+x_{1}^{2}+\ldots+x_{k}^{2}=2 x_{1} x_{1}+2 x_{2}\left(x_{1}+x_{2}\right)+\ldots+2 x_{k}\left(x_{1}+\ldots+x_{k}\right), \\
X(k)-X(k-1)=2 x_{k}\left(x_{1}+\ldots+x_{k-1}+\frac{1}{2} x_{k}\right),
\end{gathered}
$$


and the assumption on $\left\{x_{k}\right\}_{1}^{\infty}$ together imply

$$
X(k)<2 k \quad \text { and } \quad 0<X(k)-X(k-1)<2 .
$$

Let also $\gamma=1+2 / x_{1}^{2}$. Then $k \geqslant 2$ provides

$$
\frac{X(k)}{X(k-1)}=1+\frac{X(k)-X(k-1)}{X(k-1)} \quad \text { so } \quad \gamma^{-1} X(k)<X(k-1)<X(k) .
$$

By the mean value theorem applied to the square root function

$$
\begin{aligned}
\sum_{k=2}^{n} x_{k}^{p} & =\sum_{k=2}^{n}[\sqrt{X(k)}-\sqrt{X(k-1)}]^{p}<\sum_{k=2}^{n}\left[\frac{X(k)-X(k-1)}{2 \sqrt{X(k-1)}}\right]^{p} \\
& <\sum_{k=2}^{n} \frac{\gamma^{p / 2}}{2} \frac{X(k)-X(k-1)}{X(k)^{p / 2}} .
\end{aligned}
$$

The last step uses $2^{-p}[X(k)-X(k-1)]^{p}<2^{-1}[X(k)-X(k-1)]<1$.

On the other hand, application of the mean value theorem to $t \mapsto t^{-p / 2}$ produces $\xi_{k} \in[X(k-1), X(k)]$, yielding

$$
X(k-1)^{1-\frac{p}{2}}-X(k)^{1-\frac{p}{2}}=\left(\frac{p}{2}-1\right) \frac{X(k)-X(k-1)}{\xi_{k}^{p / 2}} \geqslant\left(\frac{p}{2}-1\right) \frac{X(k)-X(k-1)}{X(k)^{p / 2}} .
$$

Thus

$$
\sum_{k=2}^{n} x_{k}^{p}<\gamma^{p / 2}(p-2)^{-1} \sum_{k=2}^{n}\left[X(k-1)^{(2-p) / 2}-X(k)^{(2-p) / 2}\right]<\gamma^{p / 2}(p-2)^{-1} x_{1}^{2-p} .
$$

This gives the claimed convergence.

Now the proposition can be completed. On grounds of the standard procedure in constructing Riesz products, it is clear by identifying Fourier coefficients that if $\nu$ is constructed with the parameters $a_{k} \in[-1,1]$, then $\nu * \nu$ is also a Riesz product based on the same independent set, but with parameters $\left\{a_{k}^{2} / 2\right\}_{1}^{\infty}$. By assumption there is $\rho>0$ such that $\left\{\rho\left|a_{k}\right|\right\}$ satisfies the condition of the lemma, so one may conclude the convergence of $\sum a_{k}^{4}$. By known theory, this now says that $\nu * \nu \in L^{2}$. The proof of the proposition has been completed.

Generalized Riesz products. Both papers $[\mathrm{K}]$ and $[\mathrm{Br}-\mathrm{H}]$ consider measures arising from weak-* convergence of products of a common kind

$$
\nu=*-\lim _{m \rightarrow \infty} \prod_{j=1}^{m}\left(1-P_{j}\right)
$$

Here each $P_{j}$ is a real-valued polynomial with a particular condition on its spectrum. Write $R_{m}$ for the $m$ th partial product and $P_{j}=\sum_{k \neq 0} p_{j, k} e_{k}$. Then it is demanded that for each $m$, the spectra of

$$
e_{k} R_{m-1}, \quad \text { for all } k \text { with } p_{m, k} \neq 0,
$$

be pairwise disjoint. In particular, it follows that

$\hat{\nu}(n)$ is a finite product (over $j$ ) with at most one factor from each of the non-zero elements of $\left\{p_{j, k}\right\}_{k \neq 0}$. 
Introduce now distance functions

$$
\sigma_{p}(\mu)=\|\hat{\mu}\|_{\ell^{p}}^{p}=\sum_{k}|\hat{\mu}(k)|^{p}
$$

for $p \geqslant 1$ and measures $\mu \in M(\mathbb{T})$. In particular, $\sigma_{1}(f)=\|f\|_{A(\mathbb{T})}$ and $\sigma_{2}(f)^{1 / 2}=\|f\|_{L^{2}}$. Thus, by the spectral condition, the partial products obey

$$
\begin{aligned}
\sigma_{p}\left(R_{m}\right) & =\sigma_{p}\left(\left[1-P_{m}\right] R_{m-1}\right)=\sigma_{p}\left(R_{m-1}\right)+\sum_{k \neq 0}\left|p_{m, k}\right|^{p} \sigma_{p}\left(R_{m-1}\right) \\
& =\sigma_{p}\left(R_{m-1}\right)\left[1+\sigma_{p}\left(P_{m}\right)\right]
\end{aligned}
$$

which by induction gives

$$
\sigma_{p}\left(R_{m}\right)=\prod_{j=1}^{m}\left[1+\sigma_{p}\left(P_{j}\right)\right]
$$

Observe finally that

$$
\nu * \nu=\underset{m \rightarrow \infty}{*-\lim _{m}} R_{m} * R_{m}=\underset{m \rightarrow \infty}{*-\lim _{j=1}} \prod_{j}^{m}\left(1+P_{j} * P_{j}\right)
$$

and

$$
\sigma_{2}\left(R_{m} * R_{m}\right)=\prod_{j=1}^{m}\left[1+\sigma_{2}\left(P_{j} * P_{j}\right)\right]=\prod_{j=1}^{m}\left[1+\sigma_{4}\left(P_{j}\right)\right] .
$$

Thus it follows that $\nu * \nu \in L^{2}$ as soon as $\prod_{j=1}^{\infty}\left[1+\sigma_{4}\left(P_{j}\right)\right]<\infty$. This will be achieved for Katznelson's construction as well as for Brown's and Hewitt's.

Analysis of Katznelson's measure. In [K] the above-mentioned polynomials take the form

$$
P_{j}(\theta)=\operatorname{Re} \gamma N_{j}^{-1 / 2} \sum_{k=1}^{N_{j}} e^{i n \log n} e^{i n \lambda_{j} \theta},
$$

where $\gamma$ is a constant, the integers $\lambda_{j}$ increase fast enough to provide spectral disjointness, and $N_{j}$ satisfies (see $[\mathrm{K}]$, relation (4))

$$
2^{2(j+2)}\left\|\prod_{1}^{j-1}\left(1-P_{j}\right)\right\|_{A(\mathbb{T})}^{2}<N_{j}
$$

In particular, $N_{j}>2^{2(j+2)}$. Furthermore, it is clear that for a constant $A$

$$
\sigma_{4}\left(P_{j}\right)=2 N_{j} \cdot\left(2^{-1} \gamma N_{j}^{-1 / 2}\right)^{4}<A \cdot 4^{-j} .
$$

Thus

$$
\|\nu * \nu\|_{L^{2}}^{2}=\lim _{m \rightarrow \infty} \sigma_{2}\left(R_{m} * R_{m}\right) \leqslant \prod_{j=1}^{\infty}\left(1+A \cdot 4^{-j}\right)<\infty,
$$

which is the claimed property $\nu * \nu \in L^{2}$.

It could be recalled that the singularity of $\nu$ is based on the value $\left\|P_{j}\right\|_{2}=\sqrt{\sigma_{2}\left(P_{j}\right)}=$ $\gamma / \sqrt{2}$ for all $j \geqslant 1$. 
The measures of Brown and Hewitt. In a sense the construction of Brown and Hewitt builds on the idea of Katznelson's, so this last analysis must of necessity resemble the previous calculation.

This time the polynomials $P_{j}$ are of a more intricate nature. Among other things the non-zero coefficients $\left|p_{j, k}\right|$, for $k \geqslant 1$, of $P_{j}$, form a non-increasing finite sequence. In addition, $\left|p_{j, k}\right| \leqslant \omega(|k|)$, where $\{\omega(n)\}_{n=0}^{\infty}$ is a given admissible sequence. The details must be extracted from $[\mathrm{Br}-\mathrm{H}]$. For the present purpose, it is important that $\{\omega(n)\}_{n=0}^{\infty}$ is positive, non-increasing, and tends to zero.

In the inductive procedure of constructing $P_{m+1}$, one has to satisfy (see $[\mathrm{Br}-\mathrm{H}]$, relation (7.2.6))

$$
\omega(l)\left\|R_{m}\right\|_{A(\mathbb{T})}<2^{-m-2}
$$

by choosing $l$ large enough. Thus one finds

$$
\omega(l)<2^{-m-2} \sigma_{1}\left(R_{m}\right)^{-1}=2^{-m-2} \prod_{j=1}^{m}\left[1+\sigma_{1}\left(P_{j}\right)\right]^{-1} \leqslant 2^{-m-2} .
$$

The construction then proceeds to build $P_{j}$ from frequencies of order at least $l$, i.e., if $p_{m+1, k} \neq 0$, then $|k| \geqslant l$. Thus

$$
\left|p_{m+1, k}\right| \leqslant 2^{-m-2}, \quad \text { all } k .
$$

On the other hand, in the central result [Br-H, Thm. 6.3], the inequality (6.3.10) is equivalently providing positive constants $\alpha$ and $\beta$ with

$$
\alpha \leqslant\left\|P_{j}\right\|_{2}^{2}=\sigma_{2}\left(P_{j}\right) \leqslant \beta, \quad \text { all } j \geqslant 1 .
$$

Hence there is an estimate for all $m \geqslant 2$

$$
\sigma_{4}\left(P_{m}\right)=\sum_{k}\left|p_{m, k}\right|^{4} \leqslant 2^{-2(m+1)} \sum_{k}\left|p_{m, k}\right|^{2} \leqslant \beta \cdot 2^{-2 m-2} .
$$

The partial products can now be estimated as

$$
\left\|R_{m} * R_{m}\right\|_{2}^{2}=\sigma_{2}\left(R_{m} * R_{m}\right)=\prod_{j=1}^{m}\left[1+\sigma_{4}\left(P_{j}\right)\right] \leqslant \prod_{j=1}^{m}\left(1+\beta \cdot 2^{-2 j-2}\right) .
$$

Letting $m \rightarrow \infty$, it is clear that $R_{m} * R_{m}$ converge in $L^{2}$, so in fact $\nu * \nu \in L^{2}(\mathbb{T})$, where as before $\nu=*-\lim _{m \rightarrow \infty} R_{m}$. This was the intended property.

Concluding remarks. It is clear that not every measure in $M_{0}(\mathbb{T})$ belongs to $\operatorname{Rad} L^{1}$. A particular example is the Riesz product

$$
\mu=*-\lim _{m \rightarrow \infty} \prod_{k=2}^{m}\left[1+(\log k)^{-1} \cos 3^{k} \theta\right] .
$$

This measure is very far from being in $M_{b}$. There is even a result of Salem-Zygmund:

TheOrem [Z, page 287]. If $\mu \in M_{b}$, then

$$
\frac{\log n}{n} \sum_{k=-n}^{n}|\hat{\mu}(k)|=\mathcal{O}(1) \text {. }
$$


This decay of Fourier coefficients is in fact obtained for the function $\ell \in L^{1}$ defined by $\ell \sim \sum_{n=2}^{\infty} \frac{\cos n \theta}{\log n}$. Many more elements in $M_{b} \cap L^{1}$ may be constructed using Polya's concavity theorem.

There seem to be available only the above three constructions, for the purpose of producing singular measures in $M_{b} \cap M_{s}(\mathbb{T})$. Based on the just presented calculations, it is thus natural to ask the following question.

Problem. Is $M_{b} \backslash \operatorname{Rad} L^{1}$ non-empty?

Surrendering something to the radical, it seems more reasonable to come to terms with the next question. For $k=2$ examples are plentiful, as has just been demonstrated.

Problem. Are there other $k \geqslant 3$, such that some singular measure $\mu \in M_{b}$ has its $k$-fold self-convolution $\mu^{k} \in L^{1}(\mathbb{T})$, but still is such that $\mu^{k-1} \in M_{b} \backslash L^{1}(\mathbb{T})$ ?

Hankel-Littlewood multipliers. The intent in this main part of the paper is to produce, via Riesz products, a particular kind of Schur multipliers. To be honest, every measure on the circle group generates via its Fourier-Stieltjes coefficients a Hankel matrix which is a Schur multiplier, see Bennett's paper [Be].

The algebra of Schur multipliers $V_{2}(\mathbb{N})$ is the vector space with pointwise addition and multiplication of all $a: \mathbb{N} \times \mathbb{N} \rightarrow \mathbb{C}$ such that every operator $k \in L\left(\ell^{2}(\mathbb{N})\right)$ satisfies $\|a \cdot k\| \leqslant \alpha\|k\|$. The minimal $\alpha$ is the norm of $a$.

The decisive property of Schur multipliers (see [Be]) is that

$a \in V_{2}(\mathbb{N})$ if and only if $a: \ell^{1}(\mathbb{N}) \rightarrow \ell^{\infty}(\mathbb{N})$ can be factorized through a Hilbert space.

This suggests the following notion. For $1 \leqslant p \leqslant 2$ it is clear by the Grothendieck inequality that $T_{p} \subseteq V_{2}(\mathbb{N})$.

Definition. A function $a \in \ell^{\infty}(\mathbb{N} \times \mathbb{N})$ is called a Littlewood function of exponent $p$, where $1 \leqslant p<\infty$, if it can be decomposed as $a=b+c$, with the summands $b$ and $c$ inducing bounded operators $b: \ell^{1}(\mathbb{N}) \rightarrow \ell^{p}(\mathbb{N})$ and $c: \ell^{p^{\prime}}(\mathbb{N}) \rightarrow \ell^{\infty}(\mathbb{N})$.

The collection of such functions is denoted $T_{p}$ and it becomes a Banach algebra with the norm

$$
\|a\|_{T_{p}}=\inf \left\{\alpha>0 ; a=b+c,\|b\|_{\ell^{1} \rightarrow \ell^{p}} \leqslant \alpha,\|c\|_{\ell^{\prime \prime} \rightarrow \ell^{\infty}} \leqslant \alpha\right\} .
$$

Varopoulos introduced $T_{2}$ in [V] and the extended use with other exponents is found in Bożejko [Bo] and Wysoczański [Wy]. The same three authors observed with increasing explicitness that an equivalent norm is given by

$$
\|a\|_{T_{p}}=\inf \alpha \quad \text { with } \quad\left[\sum_{n \in E} \sum_{m \in F}|a(n, m)|^{p}\right]^{1 / p} \leqslant \alpha \max (|E|,|F|)
$$

for finite sets $E, F \subseteq \mathbb{N}$.

The reader is urged to consider this notion and Schur multipliers put in relation to the broader notion of complete boundedness. For this Pisier's monograph $[\mathrm{P}]$ is an ideal starting point, especially chapters 5 and 6 .

Definition, A sequence $c \in \ell^{\infty}(\mathbb{N})$ is a Hankel-Littlewood function of type $p$ in case the function $a(n, m)=c(n+m)$ defines a Littlewood function in $T_{p}$. The vector 
space, denoted $T_{p}^{H}$, of all these sequences, with pointwise addition and multiplication, corresponds via the Hankel matrix representation to a closed subalgebra of $T_{p}$.

It was proved by Bożejko [Bo] that a sequence $a \in \ell^{\infty}(\mathbb{N})$ belongs to $T_{p}^{H}$, where the proof in fact allows $1 \leqslant p<\infty$, if and only if

$$
\sup _{m} \sum_{k=m}^{2 m}\left|a_{k}\right|^{p}<\infty \text {. }
$$

Remark. Following Duren [D, Thm. 6.7] (or with a different proof [Bo]), the elements of $T_{2}^{H}$ correspond precisely to the multipliers from $H^{1}$ into $H^{2}$.

A variation on Bożejko's condition is more suitable to Riesz products. Consider two families of integer intervals:

$$
\begin{aligned}
I_{k}=\left[2 \cdot 3^{k}, 4 \cdot 3^{k}\right] & =\left[3^{k+1}-3^{k}, 3^{k+1}+3^{k}\right] \text { and } \\
J_{k}=\left[10 \cdot 3^{k-1}, 18 \cdot 3^{k-1}\right] & =\left[3^{k+1}+3^{k-1}, 3^{k+2}-3^{k+1}\right] .
\end{aligned}
$$

Consideration of interval length and overlap makes the following a routine task:

OBservation. $a \in T_{p}^{H}$ if and only if

$$
\sup _{k} \sum_{m \in I_{k}}\left|a_{m}\right|^{p}<\infty \quad \text { and } \sup _{k} \sum_{m \in J_{k}}\left|a_{m}\right|^{p}<\infty \text {. }
$$

Definition. Say that $\mu \in M(\mathbb{T})$ is an H-L-measure of type $p$ in case $\left.\hat{\mu}\right|_{\mathbb{N}} \in T_{p}^{H}$. The full name is of course Hankel-Littlewood measure. The collection of these measures can be denoted $M_{\mathrm{HL}^{p}}(\mathbb{T})$.

Lemma 3. Every $H$-L-measure belongs to $M_{0}(\mathbb{T})$.

Proof. Take any H-L-measure $\mu$ of type $p$. Then $\sup _{m} \sum_{k=m}^{2 m}|\hat{\mu}(k)|^{p}$ is finite, so it follows that $\mathbb{N} \cap\{k ;|\hat{\mu}(k)|>\varepsilon\}$ is a Paley set for every $\varepsilon>0$, i.e., a finite union of lacunary sets (cf. [R, page 213]). In particular, the set $\{k ;|\hat{\mu}(k)|>\varepsilon\}$ does not contain the translation of any set being the support of the Fourier-Stieltjes transform of a Riesz product. By the theorem of Host and Parreau [Ho-P], it follows that $\mu$ is a member of $M_{0}(\mathbb{T})$.

REMARK. 1) The best way to regard this is as the inclusion $B(\mathbb{N}) \cap T_{p}^{H} \subseteq B_{0}(\mathbb{N})$ for the two obvious restricted Fourier-Stieltjes algebras.

2) It is known that in general $\left.T_{p}^{H} \subseteq \mathrm{WAP}_{0}\right|_{\mathbb{N}}$, where $\mathrm{WAP}_{0}$ is the space of weakly almost periodic sequences on $\mathbb{Z}$ with zero von Neumann mean. This at least prevents contributions from discrete measures.

With the aim of producing Riesz products being H-L-measures, the lemma suggests a parameter set $a_{k}$ of complex numbers for $k \geqslant 0$ with $\left|a_{k}\right| \leqslant 1 / 2$ and $a_{k} \rightarrow 0$. The product to consider is

$$
\nu=\prod_{k=0}^{\infty}\left(1+a_{k} \exp \left[i 3^{k} \theta\right]+\overline{a_{k}} \exp \left[-i 3^{k} \theta\right]\right) .
$$

Each factor is positive and convergence is in weak-* sense. It is possible to determine precisely the conditions for membership in $T_{p}^{H}$ of this Riesz product based on the set $\left\{3^{k}\right\}$. It is for this result that the sets $I_{k}$ and $J_{k}$ were introduced as above. 
Admittedly the choice of frequencies $\left\{3^{k}\right\}$ is restrictive, but once this first analysis has been completed, the present paper will at the end of the text return to settle the question for Riesz products based on lacunary sets replacing $\left\{3^{k}\right\}$.

Every integer has a unique representation $n=\sum_{k=0}^{\infty} \varepsilon_{k} 3^{k}$ as a finite sum with $\varepsilon_{k} \in$ $\{0, \pm 1\}$. The product structure provides

$$
|\hat{\nu}(n)|^{p}=\prod_{\varepsilon_{k} \neq 0}\left|a_{k}\right|^{p}
$$

A preparatory step towards the desired characterization comes first.

Proposition 4. A Riesz product based on the frequency set $\left\{3^{k}\right\}$, and parameters $a_{k}$ with $\left|a_{k}\right| \leqslant 1 / 2$, is an $H$-L-measure of type $p$ if and only if two properties hold simultaneously:

(1) $\sup _{k}\left|a_{k}\right|^{p} A_{k}^{p}<\infty, \quad$ where $A_{k}^{p}=\prod_{l=0}^{k}\left(1+2\left|a_{l}\right|^{p}\right), \quad$ and

(2) $\sup _{k}\left|a_{k}\right|^{p} \max \left\{\left|a_{k+1}\right|^{p},\left|a_{k+2}\right|^{p}\right\} \sum_{j=1}^{k}\left|a_{j}\right|^{p} A_{j}^{p}<\infty$.

REMARK. The property $a_{k} \rightarrow 0$ is clearly a consequence of (1).

Proof. It is clear from the definition of $I_{k}$ that an index $m$ can have $\hat{\nu}(m) \neq 0$ exactly when either of four mutually exclusive possibilities occur:

( $\alpha) m=3^{k+1} \pm 3^{k}$

( $\beta) ~ m=3^{k+1}-3^{k}+3^{j}+\sum_{l=0}^{j-1} \varepsilon_{l} 3^{l}$, where $0 \leqslant j \leqslant k-1$,

$(\gamma) m=3^{k+1}+3^{k}-3^{j}+\sum_{l=0}^{j-1} \varepsilon_{l} 3^{l}$, where $0 \leqslant j \leqslant k-1$,

( $\delta) m=3^{k+1}+\sum_{l=0}^{k-1} \varepsilon_{l} 3^{l}$.

The first step is to calculate $\sum_{m \in I_{k}}|\hat{\nu}(m)|^{p}$ taking the product representation (*) into account. The case $(\alpha)$ is a triviality, whereas the contribution for the case $(\beta)$ is

$$
\left|a_{k+1}\right|^{p}\left|a_{k}\right|^{p} \sum_{j=0}^{k-1}\left|a_{j}\right|^{p} \sum_{E, F} \prod_{l \in E}\left|a_{l}\right|^{p} \prod_{l \in F}\left|a_{l}\right|^{p}=\left|a_{k+1}\right|^{p}\left|a_{k}\right|^{p} \sum_{j=0}^{k-1}\left|a_{j}\right|^{p} \prod_{l=0}^{j-1}\left(1+2\left|a_{l}\right|^{p}\right),
$$

where the inner summation is taken over all pairwise disjoint subset of $\{0, \ldots, j-1\}$. Here $E$ is the set of indices with $\varepsilon_{j}=1$ and $F$ corresponds to $\varepsilon_{j}=-1$. By symmetry, $(\gamma)$ gives the same contribution.

The case $(\delta)$ supplements a further portion

$$
\left|a_{k+1}\right|^{p} \sum_{E, F} \prod_{l \in E}\left|a_{l}\right|^{p} \prod_{l \in F}\left|a_{l}\right|^{p}=\left|a_{k+1}\right|^{p} \prod_{j=0}^{k-1}\left(1+2\left|a_{k}\right|^{p}\right)
$$

where this time $E \cup F \subseteq\{0, \ldots, k-1\}$.

Defining $A_{k}^{p}=\prod_{l=0}^{k}\left(1+2\left|a_{l}\right|^{p}\right)$ it is clear that $\sup _{k} \sum_{m \in I_{k}}|\hat{\nu}(m)|^{p}<\infty$ is equivalent to

$$
\sup _{k}\left|a_{k+1}\right|^{p} A_{k-1}^{p}<\infty \quad \text { and } \sup _{k}\left|a_{k}\right|^{p}\left|a_{k+1}\right|^{p} \sum_{j=1}^{k-1}\left|a_{j}\right|^{p} A_{j-1}^{p}<\infty .
$$

Proceeding, the membership in $J_{k}$ categorizes into four distinct categories: 
$\left(\alpha^{\prime}\right) m=3^{k+1}+3^{k-1}$ or $m=3^{k+2}-3^{k+1}$,

$\left(\beta^{\prime}\right) m=3^{k+1}+3^{k-1}+3^{j}+\sum_{l=0}^{j-1} \varepsilon_{l} 3^{l}$, where $0 \leqslant j \leqslant k-2$,

$\left(\gamma^{\prime}\right) m=3^{k+2}-3^{k+1}-3^{j}+\sum_{l=0}^{j-1} \varepsilon_{l} 3^{l}$, where $0 \leqslant j \leqslant k$,

$\left(\delta^{\prime}\right) m=3^{k+1}+3^{k}+\sum_{l=0}^{k-1} \varepsilon_{l} 3^{l}$.

Now the subcases in $\left(\alpha^{\prime}\right)$ are trivially bounded. The contribution of case $\left(\beta^{\prime}\right)$ is seen in

$$
\left|a_{k+1}\right|^{p}\left|a_{k-1}\right|^{p} \sum_{j=0}^{k-2}\left|a_{j}\right|^{p} \sum_{E, F} \prod_{l \in E}\left|a_{l}\right|^{p} \prod_{l \in F}\left|a_{l}\right|^{p}=\left|a_{k+1}\right|^{p}\left|a_{k-1}\right|^{p} \sum_{j=0}^{k-2}\left|a_{j}\right|^{p} A_{j-1}^{p}
$$

where $E$ and $F$ are disjoint subsets of $\{0, \ldots, j-1\}$. Next, the similar $\left(\gamma^{\prime}\right)$ adds correspondingly

$$
\left|a_{k+2}\right|^{p}\left|a_{k+1}\right|^{p} \sum_{j=0}^{k}\left|a_{j}\right|^{p} A_{j-1}^{p} .
$$

Lastly, the contribution from $\left(\delta^{\prime}\right)$ is

$$
\left|a_{k+1}\right|^{p}\left|a_{k}\right|^{p} A_{k-1}^{p}
$$

It follows that $\sup _{k} \sum_{m \in J_{k}}|\hat{\nu}(m)|^{p}<\infty$ is equivalent to

$$
\text { (J-bd) } \max _{l \in\{0,1\}} \sup _{k}\left|a_{k}\right|^{p}\left|a_{k+l}\right|^{p} A_{k-1}^{p}<\infty, \max _{l \in\{0,1\}} \sup _{k}\left|a_{k-1}\right|^{p}\left|a_{k+l}\right|^{p} \sum_{j=0}^{k-2}\left|a_{j}\right|^{p} A_{j-1}^{p}<\infty .
$$

Clearly the first condition in $(\mathrm{J}-\mathrm{bd})$ is weaker than its counterpart in (I-bd), which in turn is almost condition (1) in the statement of the proposition. The difference is a factor $A_{k+1}^{p} / A_{k-1}^{p}=\left(1+2\left|a_{k}\right|^{p}\right)\left(1+2\left|a_{k-1}\right|^{p}\right)$, which is uniformly bounded and hence causes no problem whatsoever.

The combination of the two second parts of (I-bd) and (J-bd) obviously says

$$
\sup _{k}\left|a_{k}\right|^{p} \max \left\{\left|a_{k+1}\right|^{p},\left|a_{k+2}\right|^{p}\right\} \sum_{j=1}^{k-1}\left|a_{j}\right|^{p} A_{j-1}^{p}<\infty .
$$

The deviation as compared to the claimed condition (2) is simply the introduction of bounded factors $A_{j}^{p} / A_{j-1}^{p}=1+2\left|a_{j}\right|^{p}$ and a, by (1), bounded term $\left|a_{k}\right|^{p} A_{k}^{p}$. These changes do not alter boundedness of the full expression, and this completes the proof.

It can be proved that in fact the condition (2) is a consequence of (1), but since it is more convenient to give both conditions an exponential form, this variation is not recorded here.

Lemma 5. Suppose the above Riesz product $\nu$ is an $H$-L-measure of type $p$. Then any $s>1$ implies $\sum_{k}\left|a_{k}\right|^{p s}<\infty$.

Corollary 6. Any such Riesz product in $M_{\mathrm{HL}^{p}}(\mathbb{T})$ is for $1 \leqslant p<2$ absolutely continuous. In fact, the Radon-Nikodym derivative is an element of $L^{2}(\mathbb{T})$. In contrast, the members in $M_{\mathrm{HL}^{p}}(\mathbb{T})$ of the above Riesz product type for $2 l \leqslant p<2 l+2$ necessarily have $\nu^{l+1}$ absolutely continuous with Radon-Nikodym derivative in $L^{2}(\mathbb{T})$, whence these $\nu$ belong to $\operatorname{Rad} L^{1}$ although are possibly singular in themselves. 
Proof. In case $1 \leqslant p<2$ one takes $s=2 / p>1$ and applies the lemma. It appears the fact that by necessity $\sum_{k}\left|a_{k}\right|^{2}<\infty$, so the condition of Brown and Moran [G-M, Thm. 7.2.1] gives the claimed absolute continuity.

On the other hand, an application of the lemma with $s=2(l+1) / p$ to $\nu \in T_{p}^{H}$ gives $\sum_{k}\left|a_{k}^{l+1}\right|^{2}<\infty$. Since the convolution power $\nu^{l+1}$ is a Riesz product on the same set $\left\{3^{k}\right\}$, but now with parameters $\left\{a_{k}^{l+1}\right\}$, an application of Brown's and Moran's theorem shows the last claim of the corollary.

Proof of Lemma 5. The type $p$ is taken fixed and and a parameter sequence $\left\{a_{k}\right\}$ satisfying the conditions in Proposition 4 is considered. Write for convenience $x_{k}=2\left|a_{k}\right|^{p}$ and $X(k)=\sum_{j=0}^{k} x_{k}$. Then $0 \leqslant x_{k} \leqslant 1$, so always $e^{x_{k}} \geqslant\left(1+x_{k}\right) \geqslant e^{x_{k} / 2}$.

Take a positive number $\alpha$ and formulate condition (1) of Proposition 4 as follows:

$$
x_{k} \prod_{j=0}^{k}\left(1+x_{j}\right) \leqslant \alpha \quad \text { for all } k \geqslant 0 .
$$

This implies that

$$
x_{k} \exp \left(\frac{1}{2} \sum_{j=0}^{k} x_{k}\right) \leqslant \alpha .
$$

The mean value theorem applied to $e^{x}$ provides real numbers $\xi_{k} \in[X(k-1), X(k)]$ such that

$$
\exp \frac{1}{2} X(k)-\exp \frac{1}{2} X(k-1)=\frac{1}{2} x_{k} \exp \frac{1}{2} \xi_{k} \leqslant \frac{1}{2} x_{k} \exp \frac{1}{2} X(k) \leqslant \frac{\alpha}{2} .
$$

It follows that

$$
\exp \frac{1}{2} X(k)=\exp \frac{1}{2} x_{0}+\sum_{j=1}^{k}\left[\exp \frac{1}{2} X(j)-\exp \frac{1}{2} X(j-1)\right] \leqslant 2+k \cdot \frac{\alpha}{2} .
$$

Hence

$$
\sum_{j=0}^{k} x_{k}=X(k) \leqslant 2 \log \left[2+\frac{\alpha k}{2}\right]
$$

It is clear that in this argument, beginning at formula $\left(1^{\prime}\right)$, it is no loss of generality to assume all $x_{k}$ to be strictly positive.

Consider now a permutation $\pi: \mathbb{N} \rightarrow \mathbb{N}$ such that $k \mapsto x_{\pi(k)}$ is non-increasing. Such permutations always exist since all $x_{k}>0$ and $x_{k} \rightarrow 0$, according to the remark after Proposition 4. Letting $j=j(k)=\max \{\pi(l) ; 0 \leqslant l \leqslant k\}$ for fixed $k$, clearly $x_{\pi(k)} \leqslant x_{j}$ and $[0, j]$ contains every $\pi(l)$ for $0 \leqslant l \leqslant k$. Therefore

$$
x_{\pi(k)} \prod_{l=0}^{k}\left(1+x_{\pi(l)}\right) \leqslant x_{j} \prod_{l=0}^{j}\left(1+x_{l}\right) \leqslant \alpha \quad \text { for each } k \geqslant 0 .
$$

Thus the argument just applied to $\left\{x_{k}\right\}$ may be performed verbatim for the altered sequence $\left\{x_{\pi(k)}\right\}$. The outcome is

$$
(k+1) x_{\pi(k)} \leqslant \sum_{j=0}^{k} x_{\pi(j)} \leqslant 2 \log \left[2+\frac{\alpha k}{2}\right] .
$$


Rewriting this as

$$
x_{\pi(k)} \leqslant \frac{2 \log [2+\alpha k / 2]}{k+1}
$$

it is routine to determine for each $s>1$ a more or less explicit, positive number $\gamma(\alpha, s)$ such that

$$
\sum_{j=0}^{\infty} x_{j}^{s} \leqslant \gamma(\alpha, s)
$$

Inserting $x_{k}=2\left|a_{k}\right|^{p}$ this is precisely the claim.

REMARK. A useful point is that given an $\alpha>0$, there is a uniform control of the Riesz products in $M_{\mathrm{HL}^{p}}(\mathbb{T})$ in the sense that any parameter sequence $\left\{a_{k}\right\}$ satisfying $\left(1^{\prime}\right)$ has also to satisfy (3) with bound only depending on $\alpha$ and $s>1$.

The Corollary 6 displays that a search for singular Hankel-Littlewood measures cannot be successful when limited to $1 \leqslant p<2$ and the present particular kind of Riesz products. For $p=2$ there is hope of a valid construction of a singular example, and in fact two different kinds will be produced in a short while.

THEOREM 7. The Riesz product $\nu$ considered above is an $H$-L-measure of type $p$ if and only if

$$
\sup _{k}\left|a_{k}\right|^{p / 2} \exp \sum_{l=0}^{k}\left|a_{l}\right|^{p}<\infty .
$$

Proof. Consider the elementary inequalities

$$
1+x \leqslant e^{x} \leqslant e^{x^{2}}(1+x) \quad \text { for all } x \geqslant 0 .
$$

Still employing the notation $x_{k}=2\left|a_{k}\right|^{p}$ it is immediate that

$$
x_{k} \prod_{j=0}^{k}\left(1+x_{k}\right) \leqslant x_{k} \exp \sum_{j=0}^{k} x_{j} \leqslant \exp \left(\sum_{j=0}^{k} x_{j}^{2}\right) x_{k} \prod_{j=0}^{k}\left(1+x_{k}\right) .
$$

According to (3) with $s=2$

$$
\sup _{k} x_{k} \prod_{j=0}^{k}\left(1+x_{k}\right) \leqslant \alpha \quad \Longleftrightarrow \quad \sup _{k} x_{k} \exp \sum_{j=0}^{k} x_{j} \leqslant \beta,
$$

with the interdependence

$$
\alpha \leqslant \beta \leqslant \alpha e^{\gamma(\alpha, 2)}
$$

If this is applied to the conditions stated in Proposition 4, the property of $\nu$ being an H-L-measure of type $p$ is equivalent to the two conditions ( since $x_{k}=2\left|a_{k}\right|^{p}$ )

$$
\begin{aligned}
& \sup _{k} x_{k} \exp X(k)<\infty, \quad \text { where } X(k)=\sum_{l=0}^{k} x_{l}, \quad \text { and } \\
& \sup _{k} \max \left\{x_{k+1}, x_{k+2}\right\} \cdot x_{k} \sum_{l=0}^{k} x_{l} \exp X(l)<\infty .
\end{aligned}
$$


The simple, real inequality $a b \leqslant\left(a^{2}+b^{2}\right) / 2$ shows the estimate

$$
2 \max \left\{x_{k} x_{k+1}, x_{k} x_{k+2}\right\} \leqslant \max \left\{x_{k}^{2}+x_{k+1}^{2}, x_{k}^{2}+x_{k+2}^{2}\right\},
$$

where the left-hand side essentially appears in (B). Thus (B) is implied by

$$
\sup _{k} \max \left\{x_{k}^{2}, x_{k+1}^{2}, x_{k+2}^{2}\right\} \sum_{l=0}^{k} x_{l} \exp X(l)<\infty .
$$

Assuming the validity of (A), the sum in this last relation can be supplemented with the terms $x_{k+1} \exp X(k+1)$ and $x_{k+2} \exp X(k+2)$ without altering the boundedness. Thus (C) is a consequence of the simplified condition

$$
\sup _{k} x_{k}^{2} \sum_{l=0}^{k} x_{l} \exp X(l)<\infty .
$$

Observe next that

$$
\exp X(l)=e^{x_{l}} \exp X(l-1) \leqslant e \cdot \exp X(l-1) .
$$

As in the proof of Lemma 5 there are numbers $\xi_{k} \in[X(k-1), X(k)]$ allowing a calculation with the mean value theorem for $e^{x}$ as the essential ingredient:

$$
\begin{aligned}
e^{-1} x_{k}^{2} \sum_{l=1}^{k} x_{l} \exp X(l) & \leqslant x_{k}^{2} \sum_{l=1}^{k} x_{l} \exp X(l-1) \leqslant x_{k}^{2} \sum_{l=1}^{k} x_{l} \exp \xi_{l} \\
& =x_{k}^{2} \sum_{l=1}^{k}[\exp X(l)-\exp X(l-1)] \\
& \leqslant x_{k}^{2} \exp X(k)=x_{k} \cdot x_{k} \exp X(k)=o(1) \cdot \mathcal{O}(1)=o(1),
\end{aligned}
$$

when applying (A) and its consequence $x_{k} \rightarrow 0$ as $k \rightarrow \infty$. The just displayed arguments show that $(\mathrm{B})$ is a consequence of $(\mathrm{A})$, whence the claimed result follows simply by taking square roots.

EXAMPLE 8. Let $a_{k}=r / \sqrt{k+\rho}$ for a positive number $r$ and where $\rho$ is so large that $a_{0} \leqslant 1 / 2$ but otherwise arbitrary. The corresponding Riesz product is a positive measure $\nu_{r}$. It is clear that

$$
\sum_{l=0}^{k} a_{l}=r \sum_{l=0}^{k}(l+\rho)^{-1 / 2}=r[2 \sqrt{k+\rho}+\omega+o(1)]
$$

for a positive constant $\omega$. Thus

$$
a_{k} \sum_{l=0}^{k} a_{l}=2 r^{2}+o(1) \quad \text { for all } k \geqslant 0,
$$

so by the theorem of Weiss' mentioned at the beginning of the paper, every measure $\nu_{r} \in M_{b}$ and so has uniformly $L^{1}$-bounded partial sums. Since $\sum_{l=0}^{\infty} a_{l}^{2}=\infty$ each $\nu_{r}$ is singular.

On the other hand

$$
\sum_{l=0}^{k} a_{l}^{2}=r^{2} \sum_{l=0}^{k}(l+\rho)^{-1}=r^{2}[\log (k+\rho)+\tau+o(1)]
$$


for a constant $\tau$. It follows that

$$
a_{k} \exp \sum_{l=0}^{k} a_{l}^{2}=r e^{r^{2} \tau}(k+\rho)^{r^{2}-1 / 2}[1+o(1)] .
$$

By the characterization in Theorem 7, this says that $\nu_{r}$ is an H-L-measure of type 2 if and only if the parameter $r \leqslant 1 / \sqrt{2}$.

The conclusion, properly recorded, is that

$$
\left.\nu_{r}=\prod_{k=0}^{\infty}\left(1+\frac{2 r \cos 3^{k} \theta}{\sqrt{k+\rho}}\right) \in M_{s}(\mathbb{T}) \cap M_{b}, \quad \text { (where } \rho \text { makes } r / \sqrt{\rho} \leqslant 1 / 2\right),
$$

is a singular measure with uniformly $L^{1}$-bounded partial sums for all $r>0$. It is a Hankel-Littlewood measure of type 2 if and only if $r \leqslant 1 / \sqrt{2}$. In particular, the set of measures $\left(M_{s}(\mathbb{T}) \cap M_{b}\right) \backslash M_{\mathrm{HL}^{2}}(\mathbb{T})$ is nonempty.

Furthermore, it is now clear that also

$$
\nu_{r}^{\prime}=\prod_{k=0}^{\infty}\left(1+(-1)^{k} \frac{2 r \cos 3^{k} \theta}{\sqrt{k+\rho}}\right)
$$

has the same properties as mentioned for $\nu_{r}$, but in addition $\nu_{r} \perp \nu_{r}^{\prime}$. This is easily seen from Brown's and Moran's theorem.

An additional idea is needed to show that for some $\nu \in M_{s}(\mathbb{T}) \cap M_{\mathrm{HL}^{p}}(\mathbb{T})$ the criterion of Weiss' fails, so it is at least conceivable that this measure does not belong to $M_{b}$. A sort of block-like description of the parameter sequence $\left\{a_{k}\right\}$ for a Riesz product will do nicely once the type is larger than two.

Example 9. First a Riesz product in $M_{\mathrm{HL}^{2}}(\mathbb{T})$, but failing Weiss' criterion, will be constructed. Consider to this end the sequence $\left\{u_{k}=2^{-1} k^{-1 / 2}\right\}_{k=1}^{\infty}$ and observe

$$
\sum_{l=1}^{k} 2 u_{l}^{2}=\frac{1}{2} \log k+\eta+o(1)
$$

whence

$$
U(k)=u_{k}^{2} \exp \sum_{l=1}^{k} 2 u_{l}^{2}=\xi k^{-1 / 2}+o(1) .
$$

A new sequence $\left\{v_{k}\right\}_{k=1}^{\infty}$ will be created by modifying $\left\{u_{k}\right\}_{k=1}^{\infty}$ at an infinitude of indices $m(j)$ to have the value $v_{m(j)}=\sqrt{j+1} / 2 \sqrt{m(j)}$. For all other indices the choice $v_{k}=u_{k}$ is applied.

Let us say that the modification is consistent as long as the inequality

$$
\sum_{j=1}^{\infty} \frac{j}{m(j)} \leqslant 2
$$

holds true.

Observe first that

$$
\sup _{k} v_{k} \sum_{l=1}^{k} v_{l} \geqslant \sup _{j} \frac{1}{4} \sqrt{j+1}\left(\frac{1}{\sqrt{m(j)}} \sum_{l=1}^{m(j)} \frac{1}{\sqrt{l}}\right)=\infty
$$

independently of the specific choices of $m(j)$. Thus Weiss' criterion always fails. 
Write for bookkeeping $\rho=\sup _{k} U(k)$, which is finite. It is decisive to record the inequality

$$
v_{k}^{2} \exp \sum_{l=1}^{k} 2 v_{l}^{2} \leqslant U(k) \exp \sum_{j=1}^{\infty} \frac{j}{2 m(j)} \leqslant e \rho
$$

for all $k$ not of the form $m(j)$. This follows from the consistency condition and the relation $v_{m(j)}^{2}=u_{m(j)}^{2}+j / 4 m(j)$. Combining $U(k)=\xi k^{-1 / 2}+o(1)$ with

$$
v_{m(j)}^{2} \exp \sum_{l=1}^{m(j)} 2 v_{l}^{2} \leqslant(j+1) \cdot U(m(j)) \exp \sum_{r=1}^{\infty} \frac{r}{2 m(r)} \leqslant(j+1) \cdot e U(m(j))
$$

it is clear that $\{m(j)\}_{j=1}^{\infty}$ can be chosen to increase so fast that the above last right-hand side always is at most $\rho$ and still the consistency condition holds. Thus, by Theorem 7 , the sequence $\left\{v_{k}\right\}$ can be used to produce a Riesz product in $M_{\mathrm{HL}^{2}}(\mathbb{T}) \cap M_{\mathrm{S}}(\mathbb{T})$ and which, as established above, does not satisfy Weiss' criterion.

A different, somewhat weaker construction is also of value. Fix $\varepsilon>0$ and choose $\alpha$ in the open interval $] 2^{-1}(1+\varepsilon)^{-1}, 2^{-1}$, whence $\left.2 \alpha(1+\varepsilon) \in\right] 1,1+\varepsilon\left[\right.$. Take $n_{k}$ to be the integer closest to $\alpha(1+\varepsilon) k^{2 \alpha(1+\varepsilon)-1}$. Then

$$
\sum_{l=1}^{k} n_{l} l^{-2 \alpha(1+\varepsilon)}=\alpha(1+\varepsilon) \log k+\mathcal{O}(1)
$$

Putting $b_{k}=k^{-\alpha}$ it is therefore clear that

$$
b_{k}^{1+\varepsilon} \exp \sum_{l=1}^{k} n_{l} b_{l}^{2(1+\varepsilon)}=\mathcal{O}(1) .
$$

Let finally the sequence $\left\{a_{k}\right\}_{k=0}^{\infty}$ consist of $n_{1}$ copies of $b_{1}$, then $n_{2}$ copies of $b_{2}$, and so on. Clearly

$$
\sup _{k} a_{k}^{1+\varepsilon} \exp \sum_{l=1}^{k} a_{l}^{2(1+\varepsilon)}=\sup _{k} b_{k}^{1+\varepsilon} \exp \sum_{l=1}^{k} n_{l} b_{l}^{2(1+\varepsilon)}<\infty .
$$

This means that the Riesz product $\nu=\prod_{k=0}^{\infty}\left(1+2 a_{k} \cos 3^{k} \theta\right)$ gives an H-L-measure of type $2(1+\varepsilon)$. On the other hand

$$
\sup _{k} a_{k} \sum_{l=1}^{k} a_{l}=\sup _{k} \alpha(1+\varepsilon) k^{-\alpha} \sum_{l=1}^{k} l^{\alpha(1+2 \varepsilon)-1}+\mathcal{O}(1)=\infty,
$$

so $\nu$ fails the criterion of Weiss'.

REMARK. These examples 8 and 9 illustrate that Weiss' condition is independent of the property determining Hankel-Littlewood measures. Whether or not the measures thus constructed belong to $M_{b}$, although failing Weiss' criterion, has not been decided by me and I have found no relevant literature.

Example 10. Consider $1 \leqslant p<2$. Thus $\frac{1}{2}<\frac{2}{2+p}$ and $1-\frac{2 p}{2+p}=\frac{2-p}{2+p}>0$.

Choosing $a_{k}=(k+1)^{-2 /(p+2)}$, the convergence of $\sum_{0}^{\infty} a_{k}^{2}$ follows. Hence the corresponding Riesz product $\nu$ is absolutely continuous with Radon-Nikodym derivative 
in $L^{2}(\mathbb{T})$. However,

$$
\sum_{l=0}^{k} a_{l}^{p}=\sum_{l=0}^{k}(l+1)^{-\frac{2 p}{2+p}}=\frac{2-p}{2+p}(k+1)^{\frac{2-p}{2+p}}+\mathcal{O}(1) \quad \text { as } k \rightarrow \infty .
$$

It follows that

$$
a_{k}^{p / 2} \exp \left(\sum_{l=0}^{k} a_{l}^{p}\right)=(k+1)^{-\frac{p}{2+p}} \exp \left[\frac{2-p}{2+p}(k+1)^{\frac{2-p}{2+p}}+\mathcal{O}(1)\right] \rightarrow \infty .
$$

In consequence, $\nu \in M_{\mathrm{ac}}(\mathbb{T}) \backslash M_{\mathrm{HL}^{p}}(\mathbb{T})$ by Theorem 7 . Clearly $\nu \in M_{\mathrm{HL}^{q}}(\mathbb{T})$ for some $p<q<2$; in fact, as soon as $2 q /(p+2)>1$.

Riesz products on more general lacunary sets. Now the time has come to deal with more general spectral sets than $\left\{3^{k}\right\}$. Consider to this end a lacunary sequence $\left\{n_{k}\right\}_{k=0}^{\infty}$ of positive integers with $n_{k+1} \geqslant 3 n_{k}$. The sequence is still dissociate, so the Riesz product construction is perfectly legal.

Main Theorem. Let $\left\{a_{k}\right\}_{k=0}^{\infty}$ be complex numbers of modulus at most $1 / 2$ and take $1 \leqslant p<\infty$. Then the Riesz product measure

$$
\kappa=\prod_{k=0}^{\infty}\left(1+a_{k} e^{i n_{k} \theta}+\overline{a_{k}} e^{-i n_{k} \theta}\right)
$$

is a Hankel-Littlewood measure of type $p$ if and only if

$$
\sup _{k}\left|a_{k}\right|^{p} \prod_{l=0}^{k}\left(1+2\left|a_{l}\right|^{p}\right)<\infty .
$$

Proof. Suppose first that $\kappa$ is an H-L-measure. Since the frequencies obey

$$
2\left(n_{k}-n_{k-1}\right) \geqslant n_{k}+3 n_{k-1}-2 n_{k-1}=n_{k}+n_{k-1},
$$

by lacunarity, the interval $\left[n_{k}-n_{k-1}, n_{k}+n_{k-1}\right]$ is included in an interval of the form $[m, 2 m]$. Thus Bożejko's characterization applied to $\left.\hat{\kappa}\right|_{\mathbb{N}}$ shows that

$$
\sup _{k \geqslant 1} \sum_{l=n_{k}-n_{k-1}}^{n_{k}+n_{k-1}}|\hat{\kappa}(l)|^{p}<\infty
$$

This interval $\left[n_{k}-n_{k-1}, n_{k}+n_{k-1}\right]$ can be used as a replacement for $I_{k}$ in the first half of the proof of Proposition 4. The calculation in that argument thus leading mutatis mutandis to $\sup _{k}\left|a_{k}\right|^{p} A_{k-1}^{p}$, and this implies ( $\left.\ddagger\right)$ in the present statement.

Conversely, the assumption that $(\ddagger)$ holds must be used to control the decisive quantity $\sup _{m} \sum_{m}^{2 m}|\hat{\kappa}(l)|^{p}$.

Let to this end $N_{k}$ and $N_{k}^{\prime}$ be the positive integers

$$
N_{k}^{\prime}=n_{k}-n_{k-1}-n_{k-2}-\ldots-n_{0} \quad \text { and } \quad N_{k}=n_{k}+n_{k-1}+\ldots+n_{0} .
$$

Clearly

$$
\operatorname{supp} \hat{\kappa} \cap \mathbb{Z}_{+}=\{m \geqslant 1 ; \hat{\kappa}(m) \neq 0\} \subseteq \bigcup_{k \geqslant 0}\left[N_{k}^{\prime}, N_{k}\right]
$$


In addition

$$
\sum_{N_{k}^{\prime} \leqslant l \leqslant N_{k}}|\hat{\kappa}(l)|^{p}=\left|a_{k}\right|^{p} \prod_{l=0}^{k-1}\left(1+2\left|a_{l}\right|^{p}\right),
$$

which is uniformly majorized by the expression in $(\ddagger)$.

Claim. $m \in\left[N_{k}^{\prime}, N_{k+1}^{\prime}\left[\right.\right.$ implies $[m, 2 m] \cap \operatorname{supp} \hat{\kappa} \subseteq\left[N_{k}^{\prime}, N_{k}\right] \cup\left[N_{k+1}^{\prime}, N_{k+1}\right]$.

Given this claim it becomes obvious that $\sup _{m} \sum_{m}^{2 m}|\hat{\kappa}(l)|^{p}$ is at most twice the number arising from the assumption of $(\ddagger)$. Thus it follows that $\kappa$ indeed is an H-L-measure if the claim can be verified.

This verification is indeed easy:

$$
N_{k+1}^{\prime}-2 N_{k}^{\prime}=n_{k+1}-3 n_{k}+n_{k-1}+\ldots+n_{0} \geqslant 0
$$

by lacunarity. This should be interpreted as

$$
N_{k}^{\prime} \leqslant m<N_{k+1}^{\prime} \quad \Longrightarrow \quad[m, 2 m] \subseteq\left[N_{k}^{\prime}, N_{k+2}^{\prime}[.\right.
$$

Since non-zero Fourier-Stieltjes coefficients of $\kappa$ are only found for frequencies in some $\left[N_{l}^{\prime}, N_{l}\right]$, the claimed result follows. This also ends the proof of the Main Theorem.

Corollary. Should condition $(\ddagger)$ hold for $p<2$, then $\kappa$ necessarily is absolutely continuous. In case $p \geqslant 2$ this condition allows singular measures, but some convolution power of $\kappa$ will be absolutely continuous. In fact, $2 l \leqslant p<2 l+1$ for some positive integer $l$ forces $\kappa^{l+1} \in M_{\mathrm{ac}}(\mathbb{T})$, while at the same time $\kappa^{l} \in M_{\mathrm{S}}(\mathbb{T})$ and $\kappa \in M_{\mathrm{HL}^{p}}(\mathbb{T})$ can be achieved.

Proof. The first claim follows by the same method as used in the first part of Corollary 6.

When $(\ddagger)$ holds for $2 l \leqslant p<2(l+1)$, then Lemma 5 may be applied with $s=2(l+1) / p$ to yield $\sum\left|a_{k}^{l+1}\right|^{2}<\infty$. Thus $\kappa^{l+1}$ is absolutely continuous with derivative in $L^{2}(\mathbb{T})$.

The particular choice $a_{k}=[2(k+\rho)]^{-1 / p}$ for $k \geqslant 0$ and where $\rho=2^{p-1}$, gives by calculations similar to Example 8 that $(\ddagger)$ holds. However, with this particular choice, the divergence of $\sum\left|a_{k}^{l}\right|^{2}$ obtains, whence this power $\kappa^{l}$ is singular.

REMARK. (i) The splitting into a detailed analysis of the ternary case and then the general lacunary case is mostly a matter of formality, but avoids some inconvenience. For instance, the intervals $\left[N_{k}^{\prime}, N_{k}\right]$ partition $\mathbb{Z}_{+}$only for $\left\{3^{k}\right\}$, as increasingly large gaps arise with the more distant (non-ternary) lacunary structures.

The main reason for the preparatory analysis is that, in general, the intervals $\left[n_{k}-\right.$ $\left.n_{k-1}, n_{k}+n_{k-1}\right]$ are not of the form $[m, 2 m]$. This would have forced a more cumbersome dissection of supp $\hat{\kappa}$ in the development of Proposition 4, which would unnecessarily have obscured the derivation of $\sup _{k}\left|a_{k}\right|^{p} A_{k-1}^{p}<\infty$, the decisive step in that result. Once the basic case has been understood, the proof of the main theorem is elementary indeed.

(ii) Examples similar to the ones produced in Examples 9 and 10 are easily constructed with the now more numerous choice of frequencies. 
Acknowledgments. I owe gratitude to Marek Bożejko in Wrocław for a fair amount of discussions on operator spaces, Schur multipliers, and other material more or less directly touching the latter part of this work.

\section{References}

[Be] G. Bennett, Schur multipliers, Duke Math. J. 44 (1977), 603-639.

[Bo] M. Bożejko, Littlewood functions, Hankel multipliers and power bounded operators on a Hilbert space, Colloq. Math. 51 (1982), 35-42.

[Br-H] G. Brown and E. Hewitt, Continuous singular measures with small Fourier-Stieltjes transforms, Adv. Math. 37 (1980), 27-60.

[D] P. Duren, Theory of $H^{p}$ Spaces, Academic Press, New York, 1970.

[G-M] C. C. Graham and O. C. McGehee, Essays in Commutative Harmonic Analysis, Springer-Verlag, New York, 1979.

[H] H. Helson, Proof of a conjecture by Steinhaus, Proc. Nat. Acad. Sci. USA 40 (1954), 205-206.

[Ho-P] B. Host and F. Parreau, Ensembles de Rajchman et ensembles de continuité, C. R. Acad. Sci. Paris 288 (1979), 899-902.

[K] Y. Katznelson, Trigonometric series with positive partial sums, Bull. Amer. Math. Soc. 71 (1965), 718-719.

[P] G. Pisier, Similarity Problems and Completely Bounded Maps, Lecture Notes in Math. 1618, Springer-Verlag, Berlin, 1996.

[R] W. Rudin, Fourier Analysis on Groups, Wiley, New York, reprinted 1990.

[V] N. T. Varopoulos, An inequality of von Neumann and an application of the metric theory of tensor products to operator theory, J. Funct. Anal. 16 (1974), 83-100.

[W] M. Weiss, On a problem of Littlewood, J. London Math. Soc. 34 (1959), 217-221.

[Wy] J. Wysoczański, Characterization of amenable groups and the Littlewood functions on free groups, Colloq. Math. 55 (1988), 261-265.

[Z] A. Zygmund, Trigonometric Series I, 2nd ed., Cambridge Univ. Press, Cambridge, 1959. 